\title{
Review
}

Clinical

nephron

Practice

\section{Renal Functional Reserve and Renal Recovery after Acute Kidney Injury}

\author{
Aashish Sharma $^{\mathrm{a}, \mathrm{c}}$ Marìa Jimena Mucino ${ }^{\mathrm{a}, \mathrm{d}}$ Claudio Ronco ${ }^{\mathrm{a}, \mathrm{b}}$ \\ anternational Renal Research Institute of Vicenza (IRRIV), and ${ }^{b}$ Department of Nephrology, Dialysis and \\ Transplantation, San Bortolo Hospital, Vicenza, Italy; ${ }^{\mathrm{C}}$ Department of Nephrology, Indraprastha Apollo Hospitals, \\ New Delhi, India; ${ }^{d}$ Intensive Care Unit, Médica Sur Clinic and Foundation, Mexico City, Mexico
}

\section{Key Words}

Acute kidney injury · Chronic kidney disease · Glomerular filtration rate $\cdot$ Hyperfiltration · Kidney attack $\cdot$ Renal functional reserve $\cdot$ Renal recovery

\begin{abstract}
Renal functional reserve (RFR) represents the capacity of the kidney to increase glomerular filtration rate (GFR) in response to certain physiological or pathological stimuli or conditions. Once baseline GFR is determined, RFR can be assessed clinically after an oral protein load or intravenous amino acid infusion. In clinical practice, baseline GFR displays variable levels due to diet or other factors. RFR is the difference between peak 'stress' GFR induced by the test (p.o. or i.v.) and the baseline GFR. In clinical scenarios where hyperfiltration is present (high baseline GFR due to pregnancy, hypertension or diabetic nephropathy, in solitary kidney or kidney donors), RFR may be fully or partially used to achieve normal or supranormal renal function. Since commonly used renal function markers, such as GFR, may remain within normal ranges until 50\% of nephrons are lost or in patients with a single remnant kidney, the RFR test may represent a sensitive and early way to assess the functional decline in the kidney. RFR assessment may become an important tool to evaluate the ability of the kidney to recover completely or partially after a kidney attack. In case of healing
\end{abstract}

with a defect and progressive fibrosis, recovery may appear complete clinically, but a reduced RFR may be a sign of a maladaptive repair or subclinical loss of renal mass. Thus, a reduction in RFR may represent the equivalent of renal frailty or susceptibility to insults. The main aim of this article is to review the concept of RFR, its utility in different clinical scenarios, and future perspective for its use.

(c) 2014 S. Karger AG, Basel

\section{Introduction}

There is a significant biodiversity among humans in the structure of the kidneys and the overall number of nephrons. This is not only governed by genetic predisposition, it is also dependent on epigenetic modifiers, such as nutritional deficiency or perinatal perturbations. This may affect the functional characteristics of the kidneys in the individual, his baseline glomerular filtration rate (GFR), and his renal functional reserve (RFR).

Targeting Recovery from Acute Kidney Injury: Round Table Conference at the 19th International Conference on Continuous Renal Replacement Therapies (Manchester Grand Hyatt, San Diego, Calif., USA, March 2-3, 2014).

\section{KARGER}

E-Mail karger@karger.com

www.karger.com/nec
C 2014 S. Karger AG, Basel

$1660-2110 / 14 / 1274-0094 \$ 39.50 / 0$
Prof. Claudio Ronco

Department of Nephrology and IRRIV

San Bortolo Hospital

IT-36100 Vicenza (Italy)

E-Mail cronco@goldnet.it 


\section{Baseline (Unstressed) GFR}

GFR is the most widely used indicator of kidney function in healthy subjects or patients with renal disease worldwide. GFR gives a rough measure of the number of functioning nephrons as it represents the sum of the filtration rates in all of them. While we can statistically identify average values for the healthy subject population, there is no such concept of normal GFR in the single individual. In fact, baseline GFR (measured in basal conditions or unstressed GFR) represents a value recorded under average dietary and hemodynamic conditions. Baseline GFR values also depend on age, sex, and body size, and are approximately 120 and $110 \mathrm{ml} / \mathrm{min} / 1.73 \mathrm{~m}^{2}$ for men and women, respectively, with considerable variation even among normal individuals [1]. GFR is remarkably stable over years, although it may change from day to day. There is an age-related decline in GFR physiologically by $0.8 \mathrm{ml} / \mathrm{min} / 1.73 \mathrm{~m}^{2} /$ year, after the age of 30 years [2-4]. It was found that the rates of decline in GFR were lower in healthy males than healthy females [5]; however, in chronic kidney disease (CKD), these rates are similar [5]. Since serum creatinine and GFR remain normal even in the presence of kidney damage until 50\% of nephrons are lost [6], baseline GFR can neither be considered a sensitive index for early detection of renal disease nor as an appropriate marker to follow up patients with renal disease. Instead, a stress test capable of exploiting the entire filtration capacity of the kidneys would probably be a more significant parameter to consider when subclinical damage is present and creatinine is still normal. This leads to the concept of RFR and its measurement as a 'stress test' for the kidney.

\section{Stress GFR and RFR}

In 1983, Bosch et al. [7] described the short- and longterm effects of protein intake on GFR by comparing normal individuals who were given either a vegetarian or high-protein diet, showing that a high-protein intake leads to a significant increase in GFR (table 1). Normal subjects display a significant increase in GFR 1 or $2 \mathrm{~h}$ after an acute protein load (1-1.2 g/kg) independently of their baseline GFR. The difference between peak or 'stress' GFR and baseline GFR describes the RFR. In 1993, Fliser et al. [8] compared the baseline and stress GFR in young and elderly healthy subjects and found that RFR was significantly lower in elderly than in young healthy individuals, but virtually all baseline GFR values of the elderly
Table 1. Comparison of heart and kidney stress states: the stress can be induced by physiological stimuli, pathological stimuli, or by a test

\begin{tabular}{|c|c|c|c|}
\hline & \multicolumn{3}{|l|}{ Organ stress state } \\
\hline & physiological & pathological & $\begin{array}{l}\text { stress } \\
\text { response test }\end{array}$ \\
\hline Cardiac & $\begin{array}{l}\text { Drugs } \\
\text { Pregnancy } \\
\text { Exercise } \\
\text { High altitude } \\
\text { Stress echo }\end{array}$ & $\begin{array}{l}\text { Hypertension } \\
\text { Hypotension } \\
\text { Sepsis } \\
\text { Anemia } \\
\text { Blood loss } \\
\text { Coronary artery } \\
\text { disease } \\
\text { VHD }\end{array}$ & $\begin{array}{l}\text { Cardiac } \\
\text { function } \\
\text { reserve }\end{array}$ \\
\hline Renal & $\begin{array}{l}\text { High protein } \\
\text { High fluid intake } \\
\text { Pregnancy } \\
\text { Aging } \\
\text { High cardiac output } \\
\text { Drugs }\end{array}$ & $\begin{array}{l}\text { Hyperfiltration } \\
\text { states } \\
\text { CKD } \\
\text { AKI } \\
\text { Congestive heart } \\
\text { failure CRS II }\end{array}$ & $\begin{array}{l}\text { Renal } \\
\text { function } \\
\text { reserve }\end{array}$ \\
\hline
\end{tabular}

In the last case, it is used to assess organ function reserve (peak vs. baseline function).

were within the normal range. In 1988, Ronco et al. [9] demonstrated differences in the GFR under baseline conditions and after acute protein load in normal and pregnant women at different stages of pregnancy without any evidence of renal disease. After acute protein load, all subjects showed a significant increase in GFR, although the difference between baseline and stress GFR values was higher in the first than in the last trimester. There was a physiological increase in baseline GFR from the first to the last trimester, which suggests a stage of physiological hyperfiltration in pregnancy. The physiologic increase in GFR during pregnancy normally results in a decrease in the concentration of serum creatinine, which falls to an

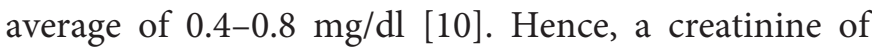
$1 \mathrm{mg} / \mathrm{dl}$ - although normal in nonpregnant women - may reflect a renal impairment in pregnancy according to creatinine-based GFR estimation.

RFR describes the capacity of the kidney to increase GFR under certain stimuli or demands. This reserve may be considered analogous to the cardiac functional reserve (as seen in cardiac stress tests). When physiological demand increases, the heart responds with an increase in cardiac output. Conceptually, the capacity to increase GFR during stress can alter the results of renal function studies. Thus, in physiological (e.g. pregnancy 


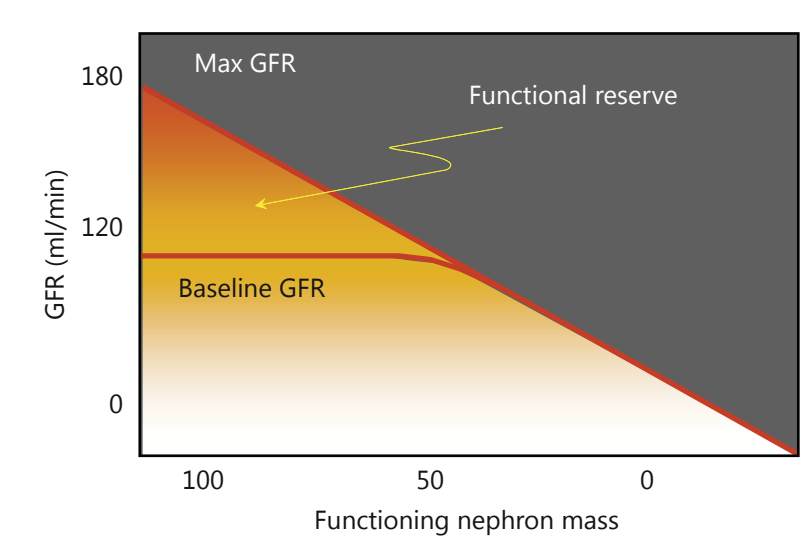

Fig. 1. RFR is the difference between the stress (peak) GFR achieved by stimulation and the baseline GFR. RFR identifies the capacity of the kidney to compensate or increase its function in states of demand or disease. Peak GFR can reach up to $180 \mathrm{ml} / \mathrm{min}$ in case of an intact nephron mass. It is reduced to approximately $120 \mathrm{ml} /$ min in case of a solitary kidney (50\% of nephron mass). In both cases, baseline GFR can result normal, but while RFR is intact in the 1 st case, it is virtually zero in the 2 nd case.

or solitary kidney) or pathological states (diabetes mellitus, nephrotic syndrome, or hypertension) [11], RFR allows to increase GFR of residual nephrons, replacing the lost function and maintaining the whole-organ GFR. Only when residual nephrons can no longer compensate for the functional loss will the changes in baseline GFR and serum creatinine occur [8] (fig. 1). On the other hand, a patient with renal disease placed on low-protein diet may have a reduction in baseline GFR unrelated to the progression of renal disease and partially restore RFR.

\section{Methods for the Estimation of RFR and Mechanisms of the Stress Test}

Several workers have described the basis of RFR after a protein load and estimation of the RFR from baseline GFR and stress GFR. In 1983, Bosch et al. [7] described the execution of renal stress tests by oral intake of $80 \mathrm{~g}$ of protein, and used baseline and stress GFR to estimate the RFR value. In 1991, De Nicola [11] executed a renal stress test to estimate RFR in treated and untreated hypertensive patients using amino acid infusion.

Numerous mechanisms have been hypothesized for the increase in GFR after protein load. Woods [12] pro- posed that the digested proteins raise plasma amino acids, which are filtered by the glomerulus and stimulate proximal tubular absorption. This and the change in the sensitivity of macula densa-sensing mechanisms cause the release of endothelium-derived relaxation factor (nitric oxide) and prostaglandins locally, causing vasodilation and increased blood flow and GFR. Also, a possible role of glucagon has been advocated. In our own laboratory, we analyzed the response to acute protein loading and we detected an increase in GFR proportional to an increase in renal blood flow indicating a constancy of filtration fraction. This observation seems to support the hypothesis that an overall increase in blood flow is the main mechanism rather than a temporary hemodynamic perturbation in the afferent/efferent arteriolar tone [unpubl. data].

\section{RFR in Disparate Clinical Settings}

There are several clinical conditions and settings where RFR is reduced either because of kidney damage or because of a physiological demand of RFR utilization (fig. 2).

\section{Pregnancy}

Pregnancy is a physiological state of hyperfiltration. GFR significantly changes during each trimester, and as such there is a significant rise in baseline GFR from the first to the last trimester. Studies done on normal pregnant women in all trimesters have shown a progressive increase in baseline GFR with a parallel reduction in RFR due to its progressive utilization. Peak GFR in normal pregnant women, however, does not change [9]. RFR measured before a pregnancy may be useful to predict and prognosticate complications in the third trimester, when the physiological demand is very high.

\section{Pathological Hyperfiltration States}

Various diseases have been associated with glomerular hyperfiltration, including diabetes mellitus, polycystic kidney disease, secondary focal segmental glomerulosclerosis with reduction in renal mass, sickle cell anemia, high-altitude renal syndrome, obesity, hypertension, or the nephrotic syndrome. Glomerular hyperfiltration may present differently in such clinical conditions. In diabetes and hypertension, for example, renal mass may be intact and baseline GFR results above normal because of efferential vasoconstriction. Hyperfiltration can also occur in residual nephrons when kidney damage has occurred and renal mass is decreased. In case of kidney damage with up
Sharma/Mucino/Ronco 
Fig. 2. Baseline and stress GFR empirically measured in different conditions. $\mathrm{CHF}=$ Congestive heart failure.

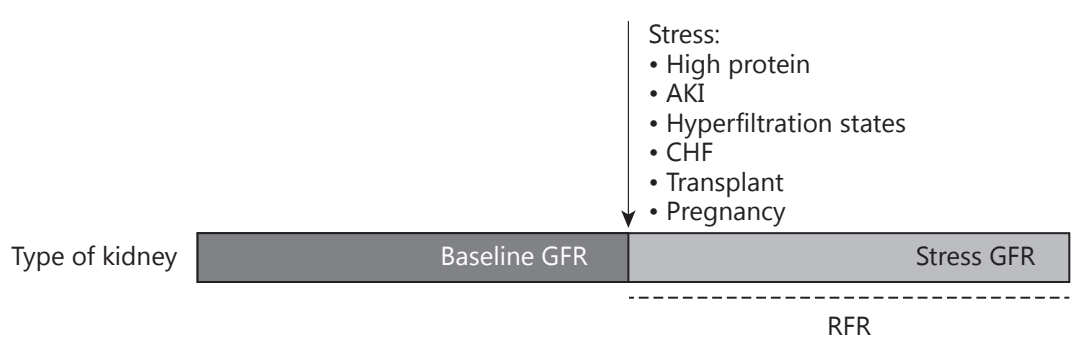

RFR

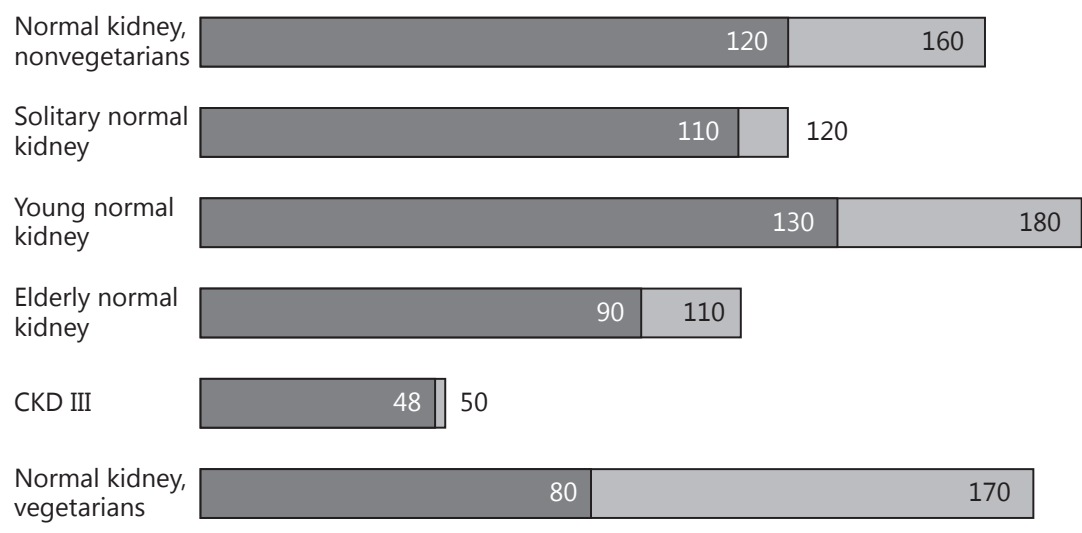

to $50 \%$ of functioning tissue, hyperfiltration in the remnant nephrons is the compensatory mechanism that ensures that GFR remains within the normal range. While in physiological states hyperfiltration is likely due to recruitment of more nephron units, in pathological states, hyperfiltration is probably due to an increase in single nephron filtration fraction. This is demonstrated by a drop in GFR when angiotensin-converting enzyme inhibitors are given to patients with CKD and kidney damage. No single definition of glomerular hyperfiltration has been agreed upon, and the pathophysiological mechanisms, which are likely to vary with the underlying disease, are not well explored. However, in pathological conditions, glomerular hyperfiltration seems likely due to a hemodynamic modification of the afferent/efferent arteriolar tone. In some cases, proteinuria may be a consequence of this mechanism.

\section{Cardiorenal Patients}

Cardiorenal syndrome type II is a well-known cause of CKD by various mechanisms like chronic hypoperfusion, necrosis, apoptosis, sclerosis, or fibrosis, but baseline GFR can remain normal for a long time due to utilization of RFR. These patients may be at risk of complications in

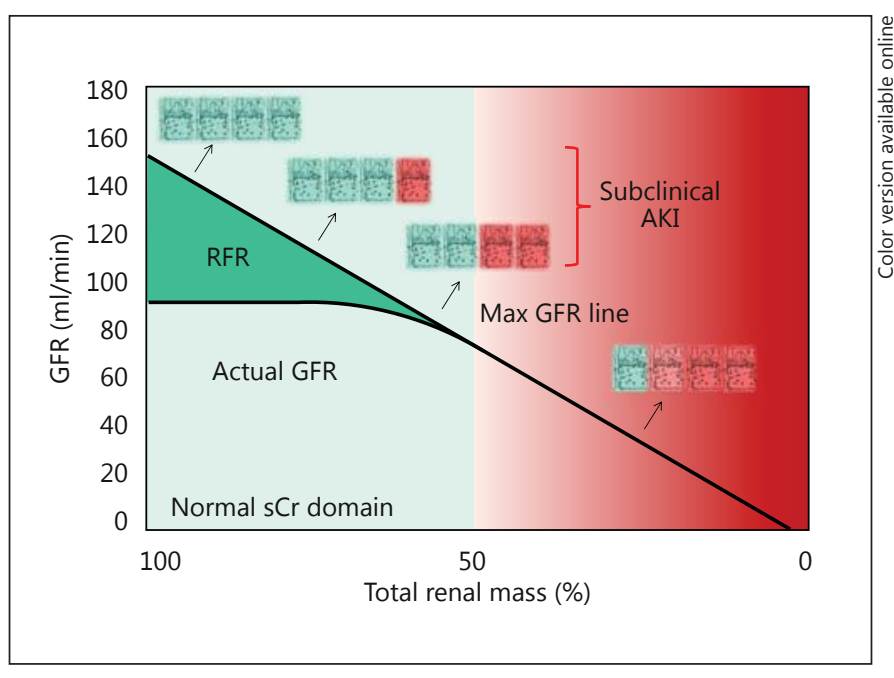

Fig. 3. AKI or kidney attack may damage a variable number of nephrons. In a subject with intact RFR, up to $50 \%$ of the nephrons can be damaged without any clinical evidence of baseline GFR reduction or increase in serum creatinine $(\mathrm{sCr})$. This remains in the subclinical domain. When further damage occurs, the syndrome will become clinically manifest. 
Fig. 4. The presence of an intact RFR and renal mass guarantees a low renal frailty or susceptibility. Even in the presence of a severe exposure (insult), the damage to the kidney may remain subclinical. On the contrary, if RFR is lost, the susceptibility of the kidney progressively increases and even in the presence of a mild exposure, the syndrome may become clinically manifest.

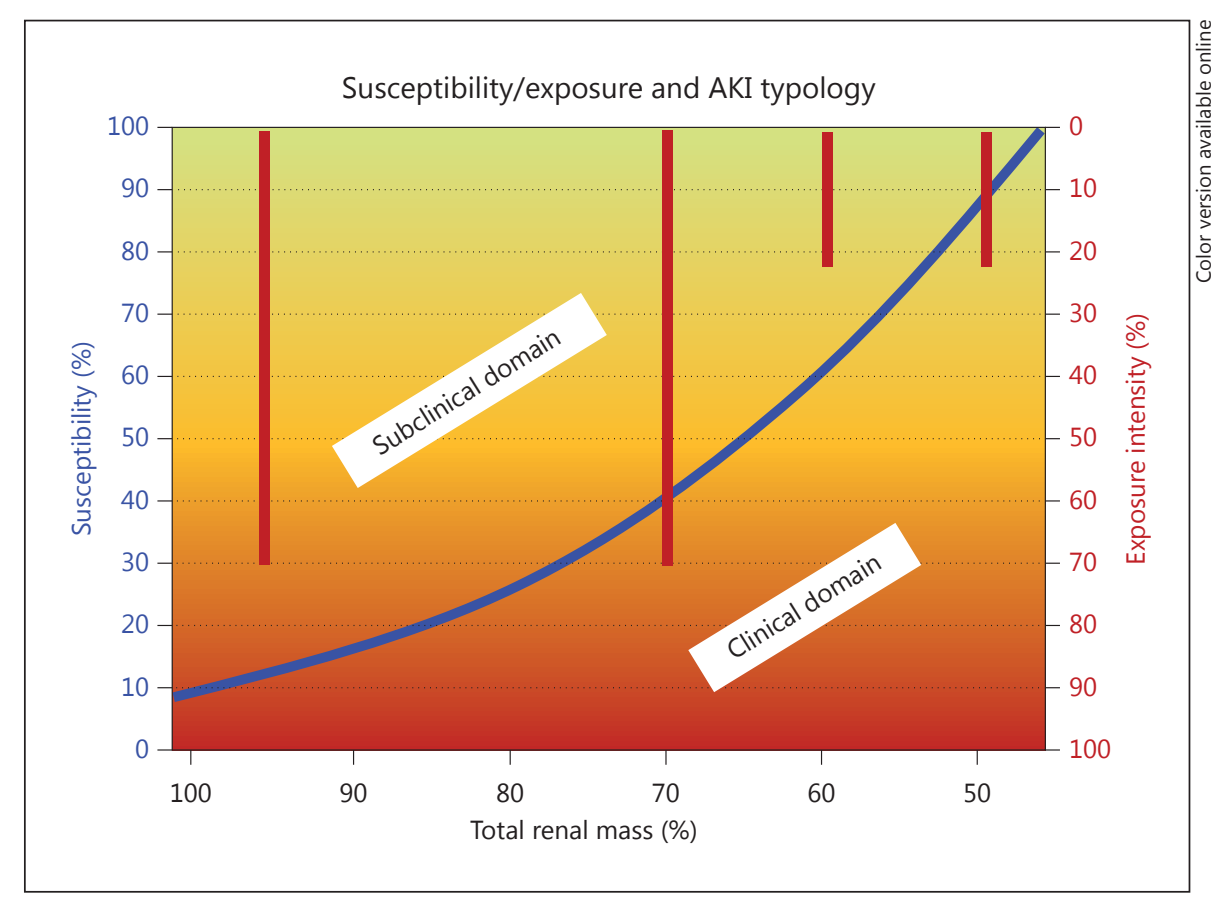

case of cardiac surgery or may have worse outcomes since they have moderate but silent CKD. Estimation of RFR in these cardiac patients and patients undergoing cardiac surgery can predict AKI and long-term outcomes.

\section{Kidney Donors}

RFR assessment can be of particular utility in determining the status of the kidneys in potential living kidney donors. Several studies controlled long-term outcomes of donors related to their RFR before donation [13]. Furthermore, a lack of RFR detected before donation may suggest to search for a more suitable donor to minimize the risks to recipient and donor.

\section{RFR and AKI: Susceptibility Index and Recovery Metrics}

Estimated GFR (eGFR) reflects a derived valued of GFR assuming that the patient is in a steady state. For this reason, eGFR is not an adequate tool to determine functional variations in AKI. However, eGFR may represent a baseline value for subsequent evaluations when the patient is followed for weeks or months. Creatinine increase due to functional changes is inherently delayed, since the kidneys have the innate ability to maintain the function by compensatory hyperfiltration in the remaining healthy nephrons irrespective of the etiology of the kidney damage or physiological demand $[6,14]$. This nephron adaptation allows for the continued clearance of plasma solute, so that serum creatinine increases only after $50 \%$ of nephrons have been lost. Thus, in a patient with intact RFR, if the insult creates a damage in less than $50 \%$ of the nephrons (hypothetically a monolateral ischemia), the syndrome may remain subclinical even if the exposure is remarkable. If part of the RFR is lost due to nephron loss in subsequent insults, the patient will display an increase in susceptibility and he may develop AKI even in the presence of an exposure of limited severity. In this framework, RFR is a good parameter to evaluate a patient's susceptibility to develop AKI (fig. 3, 4).

There is a lot of debate on how to define recovery after an acute insult. In 2004, the Acute Dialysis Quality Initiative Group [15] defined recovery as complete if the patient's level returns to within $50 \%$ of baseline serum creatinine, and partial when the patient is off renal replacement therapy but he does not attain $50 \%$ of baseline serum creatinine. No recovery was defined as persistent need for renal replacement. However, these definitions do not consider the possible role of RFR in the final evaluation of recovery. Even if creatinine levels and GFR return to baseline values, a significant reduction in RFR may have occurred and this will expose the patient to an increased susceptibility to AKI in case of future exposure (fig. 5). It has been proven that AKI may lead to nephron 


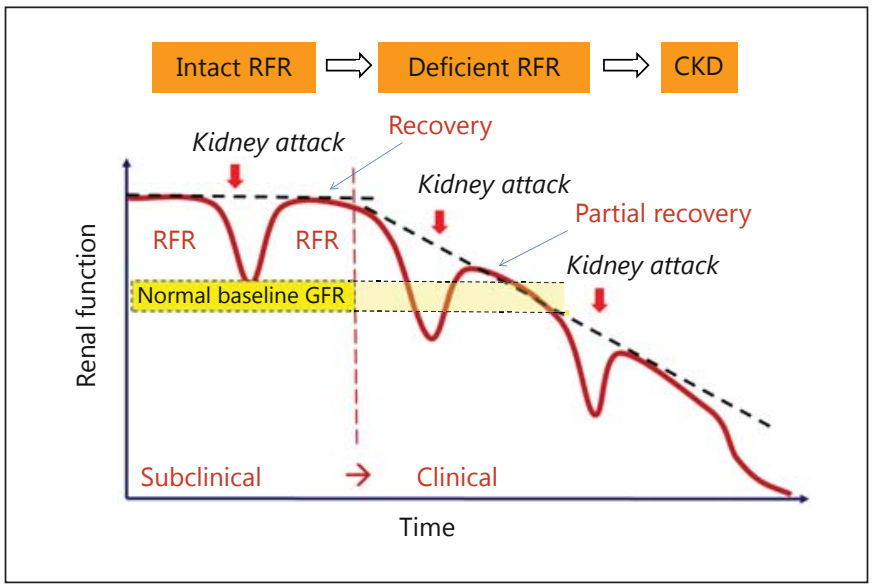

Fig. 5. If RFR is intact, an insult may remain subclinical and never displays a reduction in GFR since baseline GFR will not change. In case of a clinically evident AKI, a reduction in baseline GFR will become evident. In both cases, even if GFR returns to normal, recovery of renal function may be complete or partial. Complete or partial recovery will be documented by the assessment of RFR by a stress test. In case of partial recovery and reduced RFR, the kidney may result more susceptible to further insults and develop clinically evident AKI even in the presence of a mild exposure. A progressive defective repair will then progress towards CKD.

loss [6]; subsequently, creatinine and GFR may return to normal displaying an apparent complete recovery. In fact, the recovery may not be complete since the kidney utilizes part or whole RFR to maintain its normal function in spite of nephron loss. The association between AKI with incomplete recovery or absence of recovery and CKD has been identified recently [16]. Today, we should also consider the possible association of CKD with a reduction in RFR even if there is an apparent complete recovery. This situation is well described by the concept of subclinical AKI. Recent studies demonstrated that even AKI with apparent full recovery confers an increased risk for subsequent CKD development [16]. Risk prediction models have been developed and require further refinement and validation. The ability to identify patients with AKI recovery at high risk of developing CKD is an important clinical and research goal, which can be achieved by the assessment of RFR [16] (table 2).

\section{Conclusion}

GFR is not a fixed function and it may increase in healthy subjects in response to different stimuli. This capacity to increase the level of function depends on an intact
Table 2. Clinical data regarding RFR as a prognostic factor

\begin{tabular}{lll} 
Ref. & Clinical scenario & Outcome \\
\hline 17 & $\begin{array}{l}\text { Normotensive } \\
\text { systemic sclerosis } \\
\text { with normal renal }\end{array}$ & $\begin{array}{l}\text { After } 5 \text { years, reduction in creatinine } \\
\text { clearance, microalbuminuria, or } \\
\text { sunctemic hypertension }\end{array}$ \\
$\begin{array}{l}\text { surinary and no } \\
\text { abnormalities }\end{array}$ & $\begin{array}{l}\text { Patients with abnormal RFR showed } \\
\text { a significantly higher reduction in } \\
\text { creatinine clearance than patients } \\
\text { with normal RFR } \\
\end{array}$ \\
& $\begin{array}{l}\text { No patient with normal RFR } \\
\text { developed microalbuminuria of high } \\
\text { blood pressure }\end{array}$
\end{tabular}

18 CKD patients at Baseline and stimulated GFR

various stages following amino acid infusion, renal reserve was expressed as percentage of GFR

Renal reserve falls from $23.4 \%$ in normal controls to $6.7 \%$ in stage 4 CKD

Renal reserve may be exhausted even with a normal basal GFR

19 RFR increase in Midterm borderline hypertensive normotensive and pregnant patients failed to increase hypertensive GFR as much as normotensive pregnant women pregnant women did after a protein challenge

20 Obese hypertensive Renal reserve was lower in obese patients were compared with 9 lean hypertensives hypertensives when compared with that of lean hypertensives

nephron mass and describes RFR. Thus, baseline GFR does not describe the entire functional capacity of the kidneys and a stress test is required to quantify the functional reserve of the organ. At the same time, even if a complete recovery after AKI is observed, a significant nephron loss may have occurred with a consequent reduction in RFR. In this case, the stress test is the only possibility to unveil incomplete recovery and initial progression towards CKD. Based on the susceptibility/exposure model, we might speculate that more than $80 \%$ of $\mathrm{AKI}$ is occurring in predisposed individuals with reduced RFR, and they should therefore be defined as 'acute on chronic kidney injury'.

In conclusion, a kidney stress test devoted to assess RFR may be useful

(a) To assess and prognosticate a silent renal disease in high-risk groups, e.g., hypertension, diabetes mellitus, polycystic kidney disease, solitary kidney, and CKD, or in patients facing an aggressive intervention and dangerous exposures. 
(b) To evaluate living kidney donors to make sure no problem will occur to the donor and the recipient after transplantation.

(c) To make a clinical risk assessment in individuals undergoing high-risk surgeries, e.g., cardiac surgery, contrast imaging techniques, or other exposures (fig. 3, 4) [16].

(d) To evaluate the level of recovery after AKI.

More studies in different cohorts and stages of the population are required to more clearly understand the long-term benefits of assessing RFR. A better and refined technique has to be developed in order to make RFR assessment easier and practical in clinical routine.

\section{Disclosure Statement}

All authors declare no conflict of interest.

\section{References}

1 Stevens LA, Coresh J, Greene T, Levey AS: Assessing kidney function - measured and estimated glomerular filtration rate. $\mathrm{N}$ Engl J Med 2006;354:2473-2483.

$\checkmark 2$ Davies DF, Shock NW: Age changes in glomerular filtration rate, effective renal plasma flow, and tubular excretory capacity in adult males. J Clin Invest 1950;29:496-507.

3 Wesson LG Jr: Renal hemodynamics in physiological states; in Wesson GL: Physiology of the Human Kidney. New York, Grune \& Stratton, 1969, pp 96-108.

4 Macías-Núñez JF, López-Novoa JM: Physiology of the healthy aging kidney; in MacíasNúñez JF, Cameron JS, Oreopoulos DM (eds): The Aging Kidney in Health and Disease. New York, Springer, 2008, pp 93-112.

5 Xu R, Zhang LX, Zhang PH, Wang F, Zuo L, Wang HY: Gender differences in age-related decline in glomerular filtration rates in healthy people and chronic kidney disease patients. BMC Nephrol 2010;23:11-20.

6 Liu KD, Brakeman PR: Renal repair and recovery. Crit Care Med 2008;36:S187-S192.

7 Bosch JP, Saccaggi A, Lauer A, Ronco C, Belledonne M, Glabman S: Renal functional reserve in humans. Effect of protein intake on glomerular filtration rate. Am J Med 1983;75: 943-950.
8 Fliser D, Zeier M, Nowack R, Ritz E: Renal functional reserve in healthy elderly subjects. J Am Soc Nephrol 1993;3:1371-1377.

-9 Ronco C, Brendolan A, Bragantini L, Chiaramonte S, Fabris A, Feriani M, Dell Aquila R, Milan M, Mentasti P, La Greca G: Renal functional reserve in pregnancy. Nephrol Dial Transplant 1988;3:157-161.

10 Fischer MJ: Chronic kidney disease and pregnancy: maternal and fetal outcomes. Adv Chronic Kidney Dis 2007;14:132-145

11 De Nicola L, Blantz RC, Gabbai FB: Renal functional reserve in treated and untreated hypertensive rats. Kidney Int 1991;40:406-412.

12 Woods LL: Mechanisms of renal haemodynamic regulation in response to protein feeding. Kidney Int 1993;44:659-675.

13 Rooka M, Hofkerb HS, van Sona WJ, Homan van der Heidea JJ, Ploegb RJ, Navisa GJ: Predictive capacity of pre-donation GFR and renal reserve capacity for donor renal function after living kidney donation. Am J Transplant 2006;6:1653-1659.

14 Helal I, Fick-Brosnahan GM, Reed-Gitomer B, Schrier RW: Glomerular hyperfiltration: definitions, mechanisms and clinical implications. Nat Rev Nephrol 2012;8:293-300.

15 Bellomo R, Ronco C, Kellum JA, Mehta RL, Palevsky P; Acute Dialysis Quality Initiative Workgroup: Acute renal failure - definition, outcome measures, animal models, fluid therapy and information technology needs: the Second International Consensus Conference of the Acute Dialysis Quality Initiative (ADQI) Group. Crit Care 2004;8:R204-R212.
6 Heung M, Chawla LS: Predicting progression to chronic kidney disease after recovery from acute kidney injury. Curr Opin Nephrol Hypertens 2012;21:628-634.

17 Livi R, Guiducci S, Perfetto F, Ciuti G, Grifoni E, Conforti L, Galluccio F, Moggi Pignone A, Matucci Cerinic M: Lack of activation of renal functional reserve predicts the risk of significant renal involvement in systemic sclerosis. Ann Rheum Dis 2011;70: 1963-1967.

18 Barai S, Gambhir S, Prasad N, Sharma RK, Ora M: Functional renal reserve capacity in different stages of chronic kidney disease. Nephrology 2010;15:350-353.

19 Heguilén RM, Liste AA, Bellusci AD, Lapidus AM, Bernasconi AR: Renal response to an acute protein challenge in pregnant women with borderline hypertension. Nephrology 2007;12:254-260.

20 Pecly IM, Genelhu V, Francischetti EA: Renal functional reserve in obesity hypertension. Int J Clin Pract 2006;60:1198-1203.

21 Ronco C, Rosner MH: Acute kidney injury and residual renal function. Crit Care 2012; 16:144. 